Supporting Information

\title{
Covalently Modulated and Transiently Visible Writing: Rational Association of Two Extremes of Water Wettabilities
}

Supriya Das, ${ }^{\dagger}$ Ravi Kumar, ${ }^{\ddagger}$ Dibyangana Parbat, ${ }^{\dagger}$ Sylwia Sekula-Neuner, ${ }^{\ddagger}$ Michael Hirtz,,${ }^{*}$ and Uttam Manna*,†

\footnotetext{
${ }^{\dagger}$ Department of Chemistry and Centre for Nanotechnology, Indian Institute of Technology-Guwahati, Kamrup, Assam 781039, India. E-mail: umanna@iitg.ac.in

${ }^{\ddagger}$ Institute of Nanotechnology (INT) \& Karlsruhe Nano Micro Facility (KNMF), Karlsruhe Institute of Technology (KIT), Hermann-von-Helmholtz-Platz 1, 76344 Eggenstein-Leopoldshafen, Germany. E-mail: michael.hirtz@kit.edu
} 

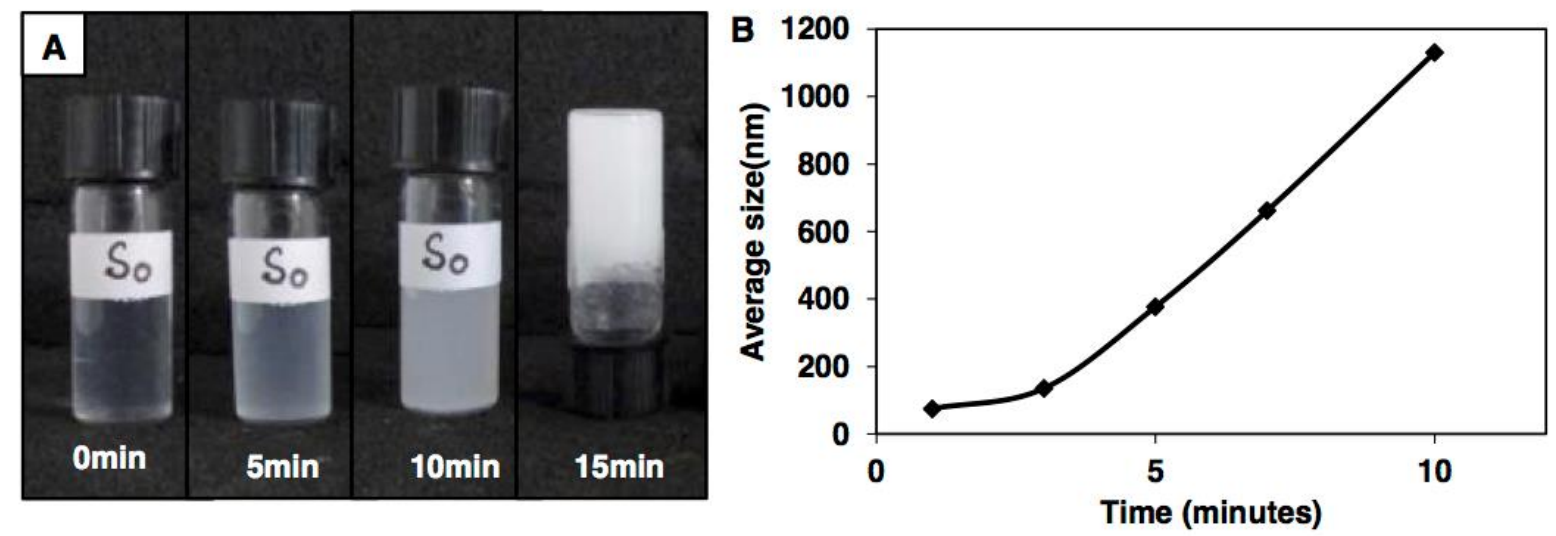

Figure S1. (A) Digital image accounts change in appearance of the reaction mixture of BPEI/5Acl in heptanol over the time. (B) DLS study accounting the change in size of the polymeric nanocomplex in the reaction mixtures over different time (e.g.; 1 minute, 3 minutes, 5 minutes, 7 minutes, 10 minutes).
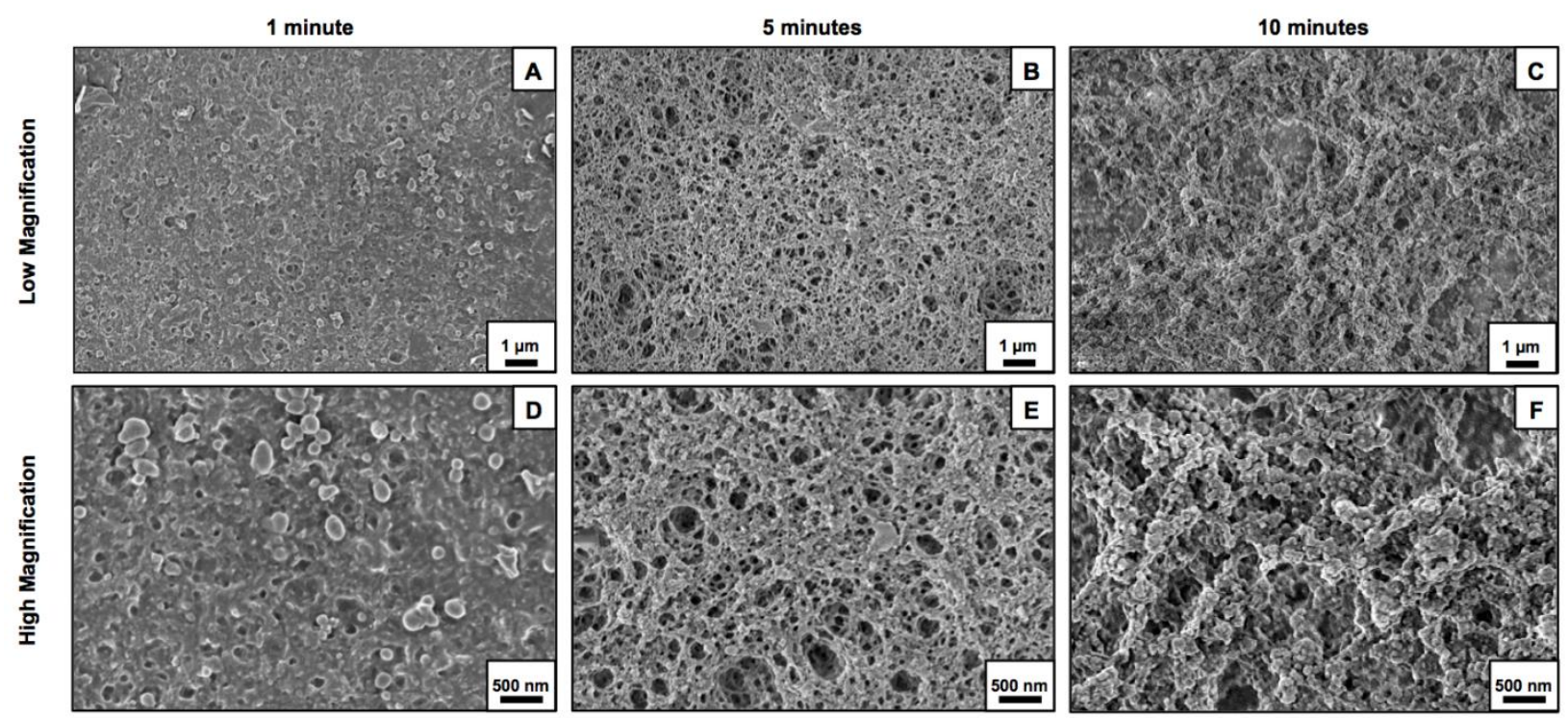

Figure S2. (A-F) FESEM images of the polymeric dip coatings that are prepared by immerging the selected substrate in reaction mixture for 1 minute (A,D), 5 minutes (B,E) and10 minutes $(\mathrm{C}-\mathrm{F})$, in both low $(\mathrm{A}, \mathrm{B}, \mathrm{C}$ scale bar $=1 \mu \mathrm{m})$ and high $(\mathrm{D}, \mathrm{E}, \mathrm{F}$ scale bar $=0.5 \mu \mathrm{m})$ magnifications. 


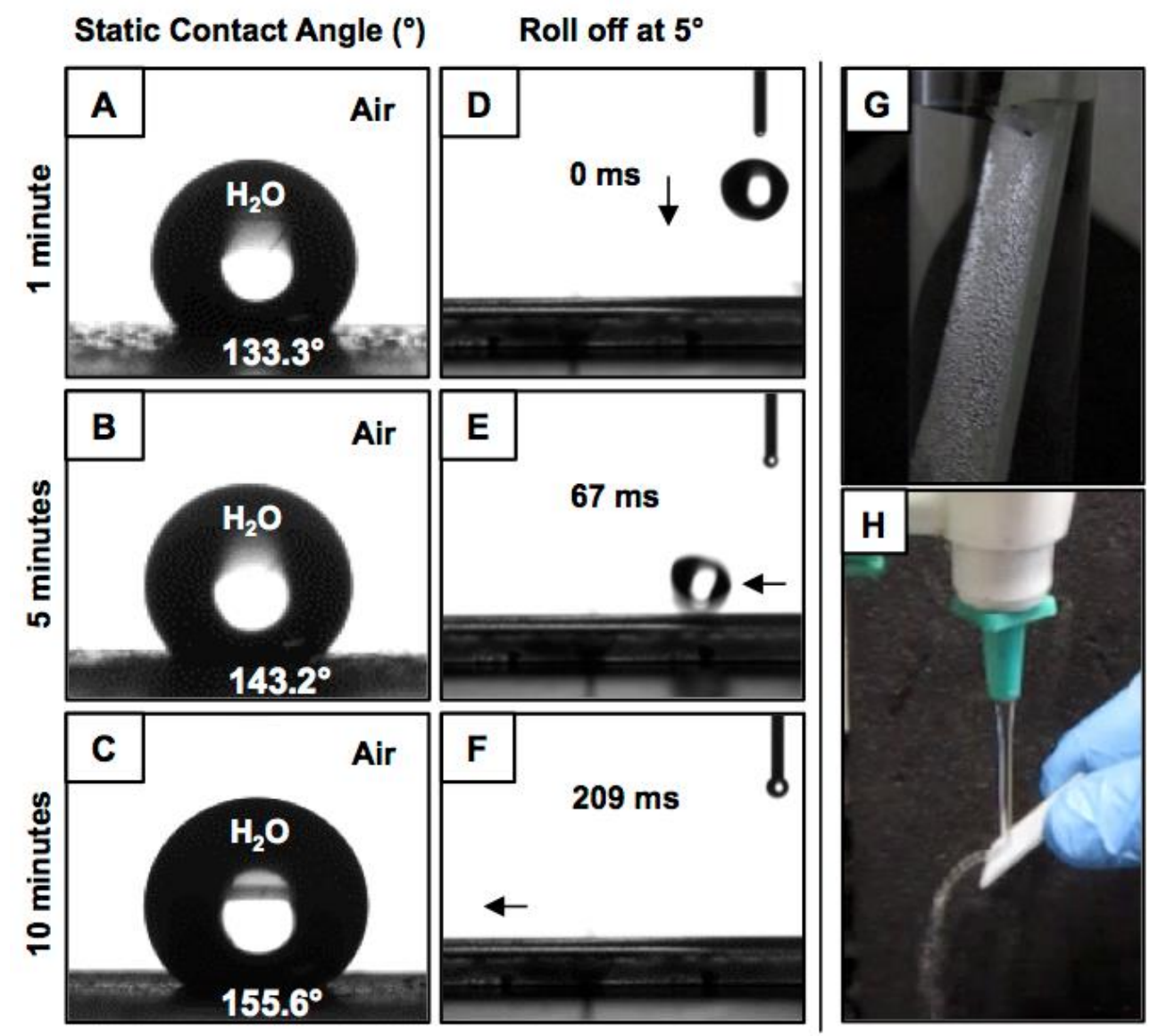

Figure S3. (A-C) Static contact angles (CA) of the beaded water droplet on the octadecylamine (ODA) treated polymeric coatings that were prepared by immersing the glass slide for 1 minute (A), 5 minutes (B) and 10 minutes (C). (D-F) Contact angle images illustrating rolling of beaded water droplet on the tilted $\left(5^{\circ}\right)$ superhydrophobic dip coating. $(\mathrm{G})$ Digital image showing the shiny interface of water/polymeric coating, which revealed the presence of metastable trapped air in the polymeric dip coating that was prepared by prepared by immersing the glass slide for 10 minutes and post modified with ODA. (H) Digital photograph of same polymeric coating that was exposed to the jet of water, where liquid water bounced away after hitting the superhydrophobic surface. 


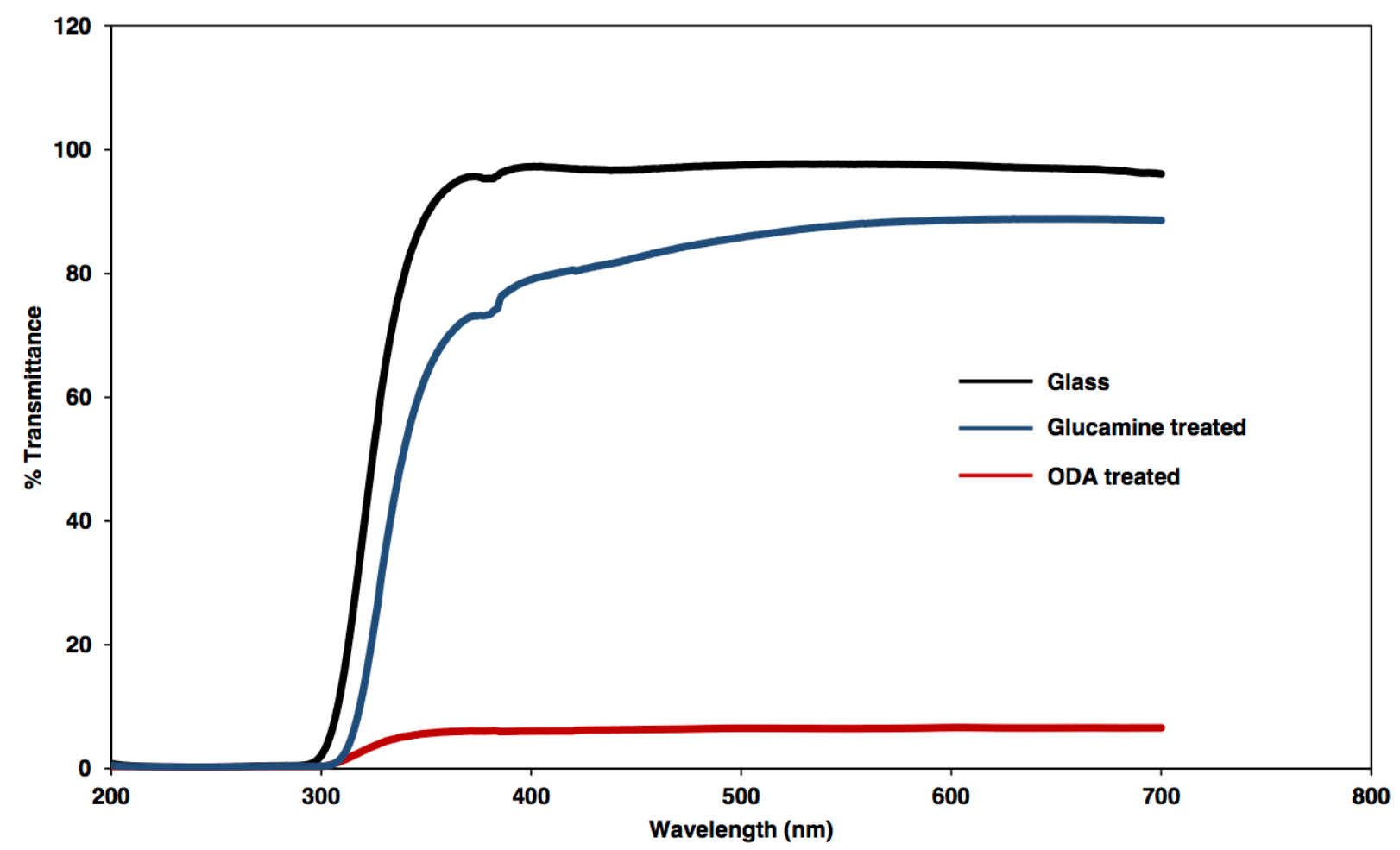

Figure S4. The plot comparing the underwater optical transparency of the bare glass (black curve) with polymeric dip coated glass substrates that are post modified with octadecylamine (red curve) and D-glucamine (blue curve) treated.
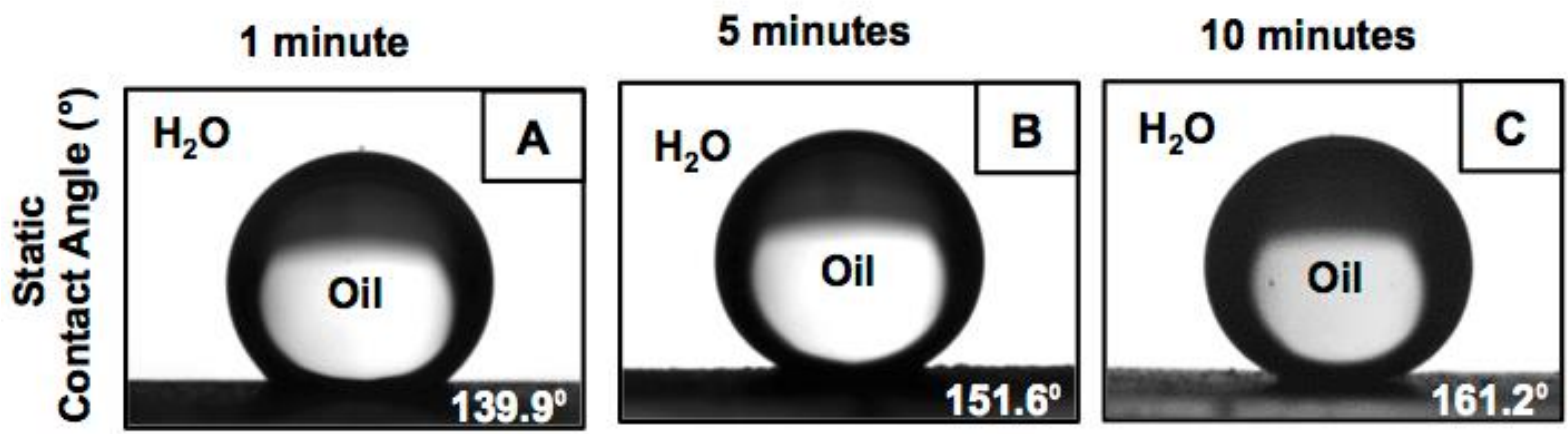

Figure S5. (A-C) Static contact angles (underwater) of beaded oil droplets on the glucamine functionalized polymeric coatings that are prepared by immersing the glass substrate in reaction solution for 1 minute (A), 5 minutes (B), 10 minutes (C). 

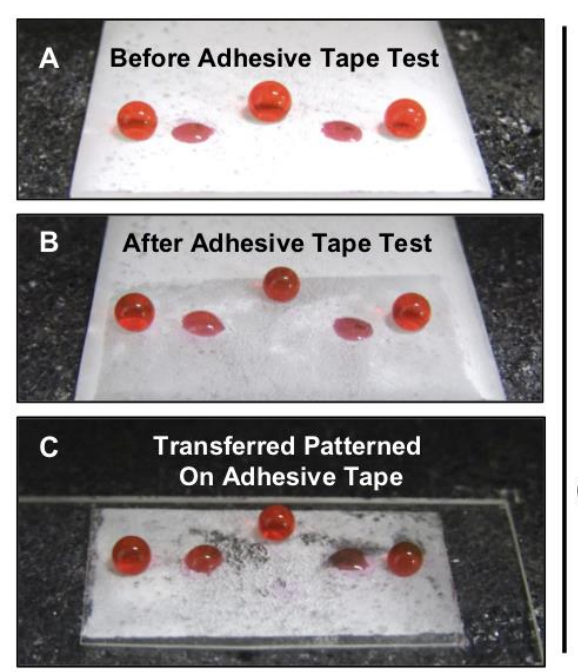

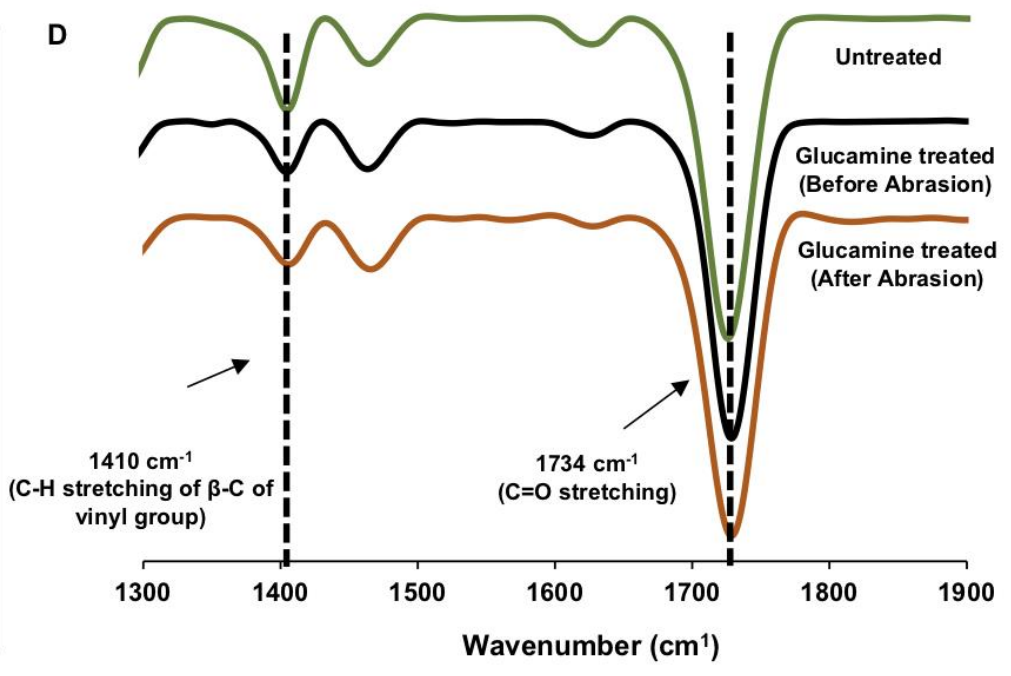

Figure S6. (A-C) Digital images showing the retention of the circular hydrophilic pattern (glucamine treated) prepared on the superhydrophobic (post-treated with ODA) dip coated glass substrates (A), after performing the adhesive tape test on the chemically modulated pattern interface (B). During the course of adhesive tape peeling test, the top portion of the polymeric coating is randomly cleaved, and was transferred onto the adhesive tape (C), which resulted in exposure to the interiors of the polymeric coating. However, the patterned water wettability (glucamine treated spots are hydrophilic, other parts treated with ODA superhydrophobic) remained intact in the freshly exposed interiors of the polymeric coating, revealing the three dimensional and inherently durable nature of the patterned interface. D) Representing IR spectra of polymeric coating before (black line) and after (and orange line) physical abrasion of the glucamine-treated polymeric coating, where both the IR spectra were compared with IR spectrum of untreated polymeric coating (green line). 

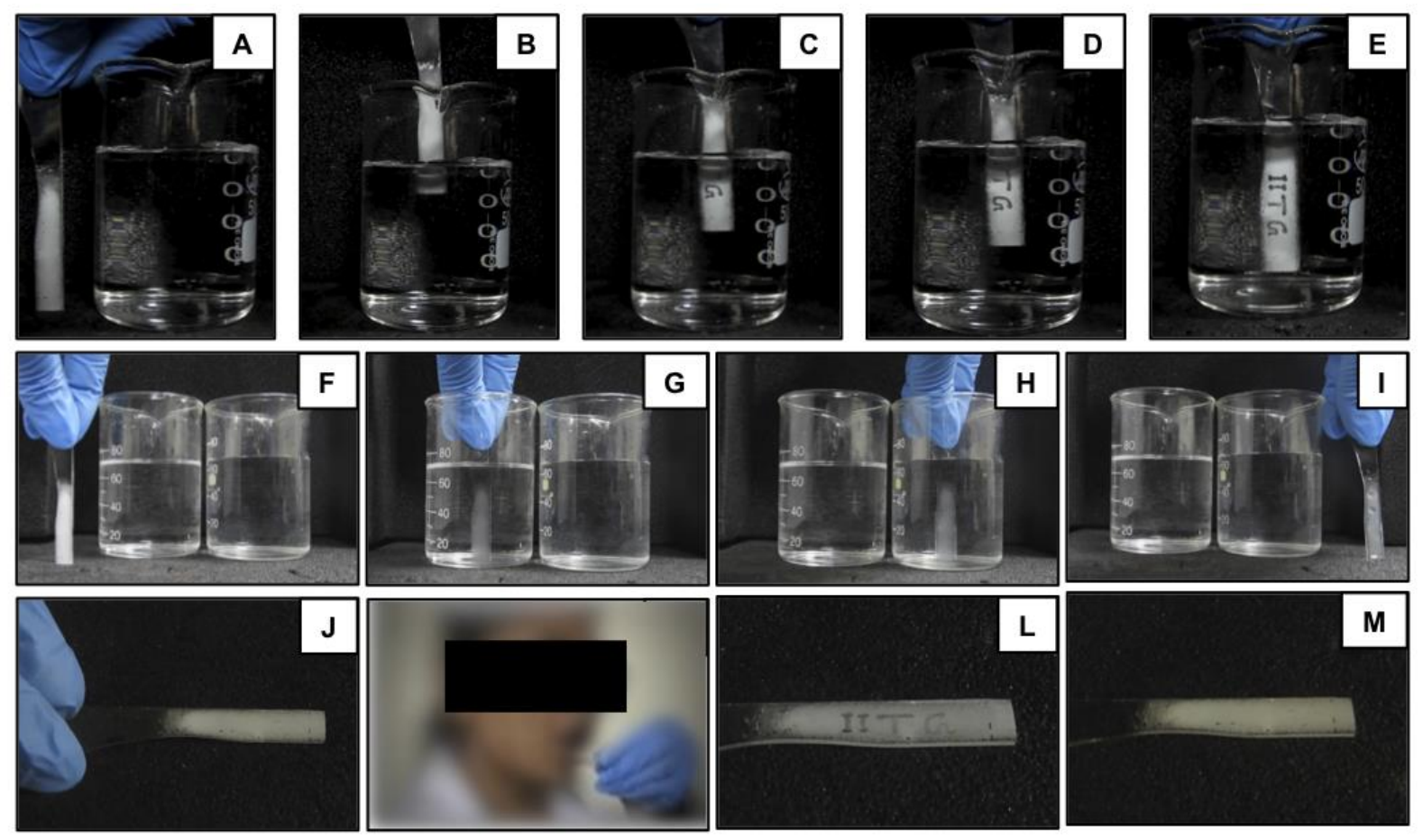

Figure S7. (A-E) Digital images illustrating the appearance of visible hand writing (in air, A), once the patterned interface is exposed to liquid water (A-E). Whereas no such visibility is observed if the patterned interface is exposed to ethanol (F-G), prior to submerging in water (H-I), the exposure of ethanol removes the metastable trapped air. The selective entrapment of metastable trapped air is crucial for the appearance of invisible pattern. (J-M) Digital images illustrating the appearance of invisible hand-writing $(\mathrm{J})$ on exposure to mouth's mist (K-L), and this pattern was disappeared on air drying of condensed aqueous phase (M). 

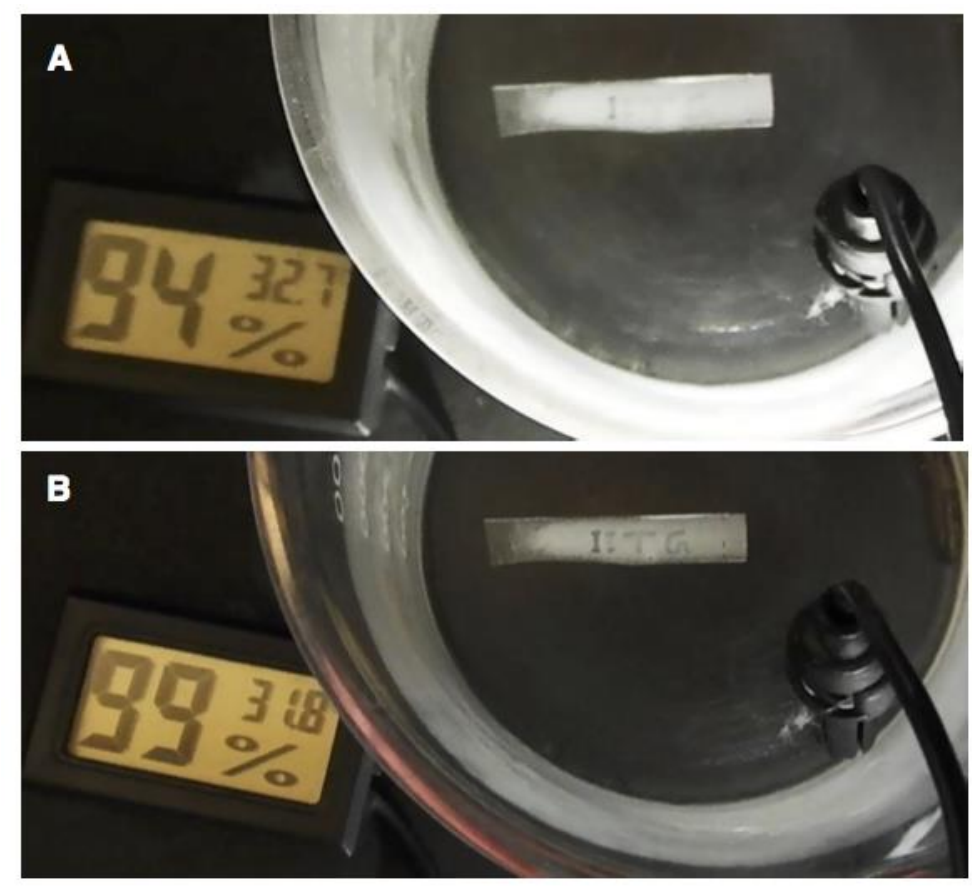

Figure S8. (A-B) Digital images accounting the change in visibility of the chemically modulated patterned interface in humid air. The visibility of the pattern is clear (B) with 99\% humid air, whereas the appearance of the pattern is faint in $94 \%$ humid air (A). 

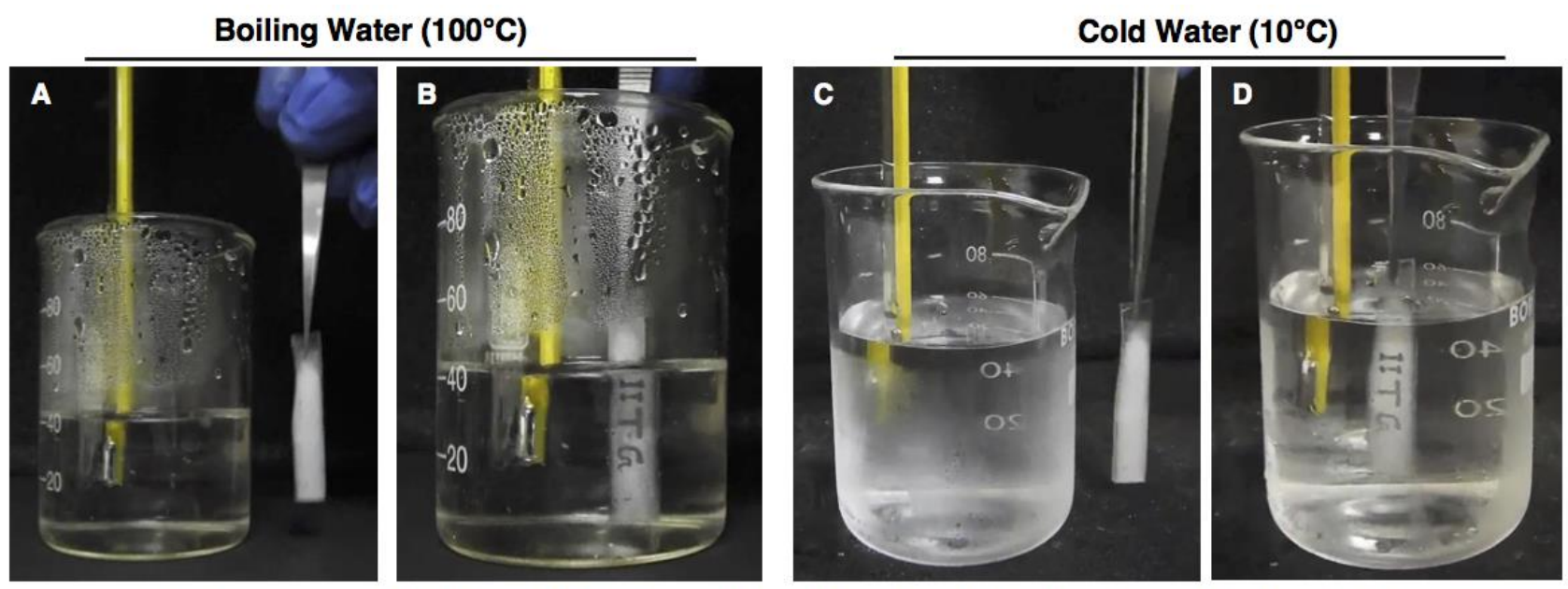

Performance of Patterned Interface After Abrasive Adhesive Tape Peeling Test

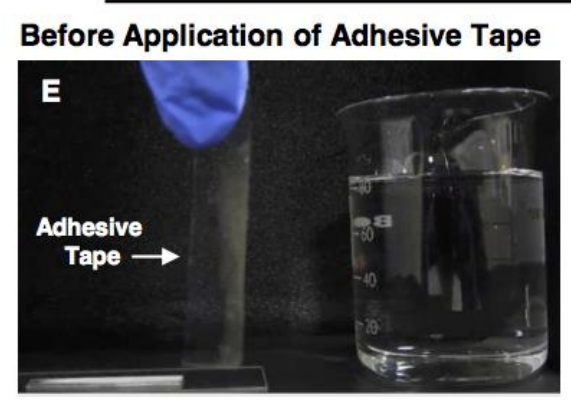

Adhesive Tape Peeling Process
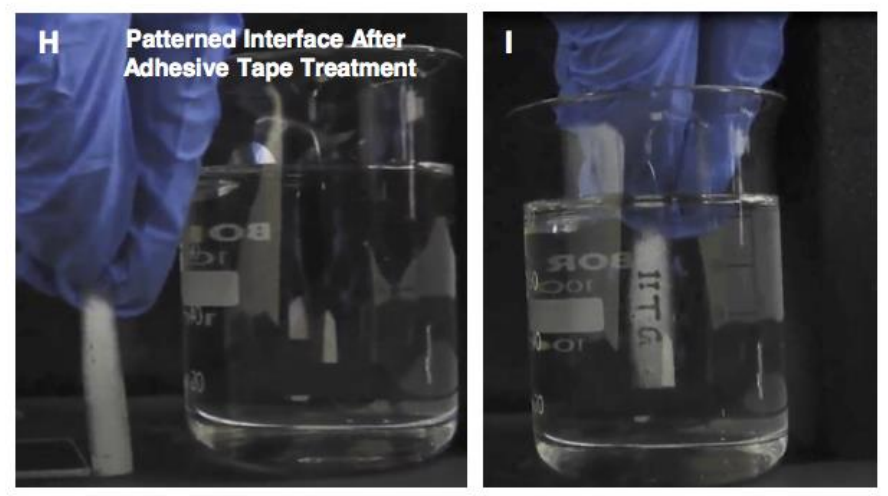

Figure S9. (A-D) Demonstrating the performance of an invisible chemically patterned interface at high $\left(100^{\circ} \mathrm{C}\right.$; A-B) and low $\left(10^{\circ} \mathrm{C}\right.$; C-D) temperatures. (E-I) Digital images illustrating both adhesive tape tests (E-G) and the performance (H-I) of the physically abraded polymeric patterned interfaces under water. During the adhesive tape peeling process, the top portion of the polymeric coating is physically abraded $(\mathrm{G})$. However, the invisible chemically modulated pattern remains intact and the physically abraded pattern is visible under water (I). This impeccable durability of the invisible patterned is attributed to the existence of threedimensional patterned wettability in the polymeric coating as evident from Fig. S6. 


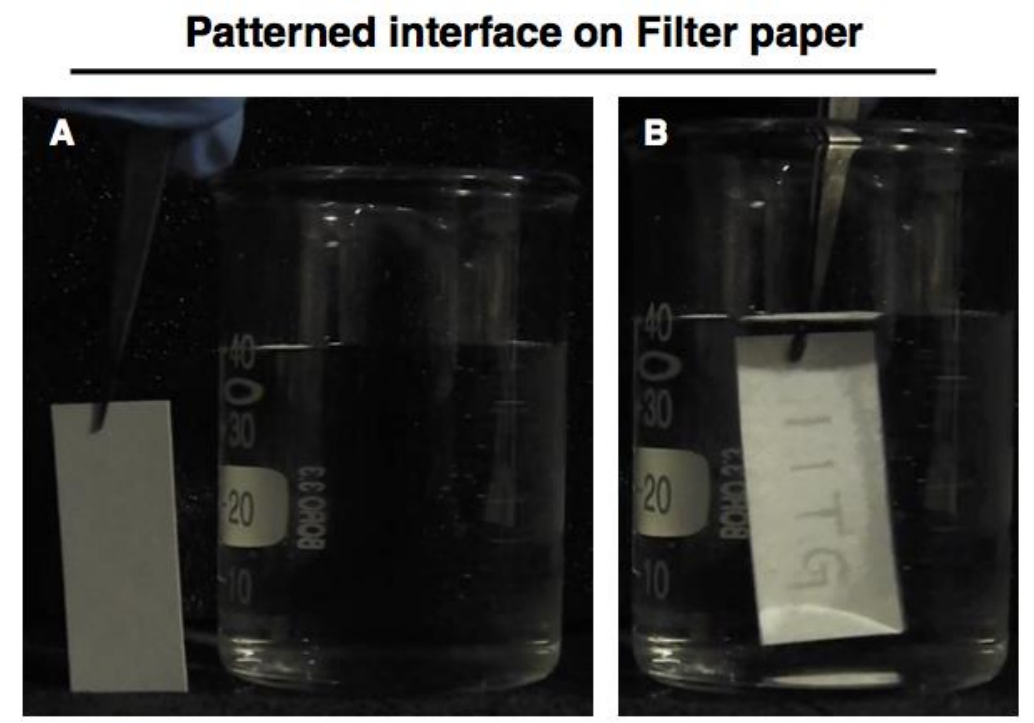

Figure S10. (A-B) Digital images accounting the performance of the invisible chemically modulated patterned interface on a filter paper. The pattern is invisible in air (A) on the polymeric coating on an opaque substrate but becomes visible under water (B), likely due to selective displacement of external metastable trapped air from glucamine treated regions. 


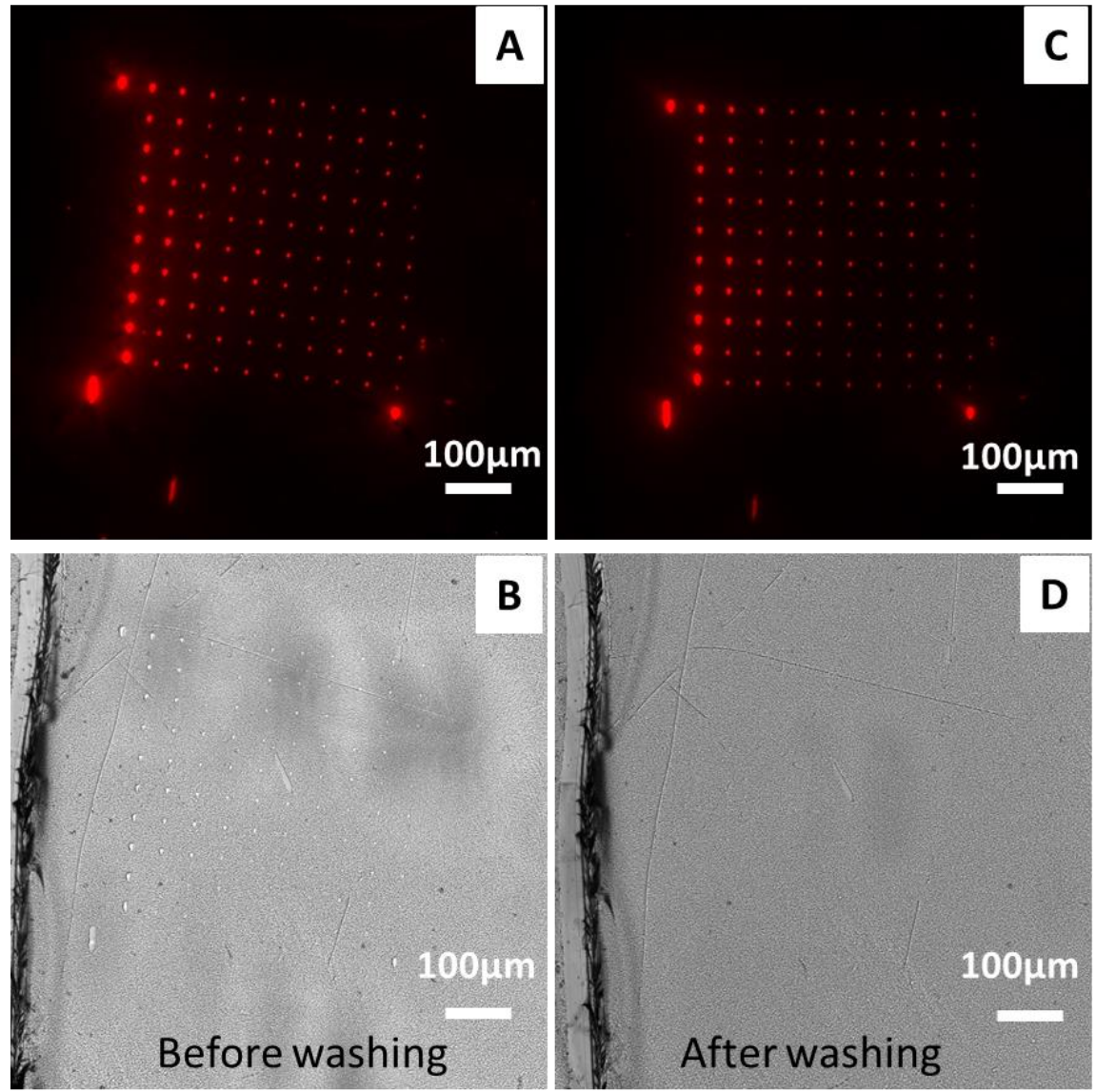

Figure S11. The microscopic images of 10x10 matrix patterned by $\mu$ CS with STV_cy3 ink on amine reactive polymeric dip coating substrate. (A) (cy3 channel) and (B) (BF) are images before washing, (C) and (D) are after washing. The pattern visible only in the cy3 channel after washing confirm successful immobilization of a thin film of protein. 

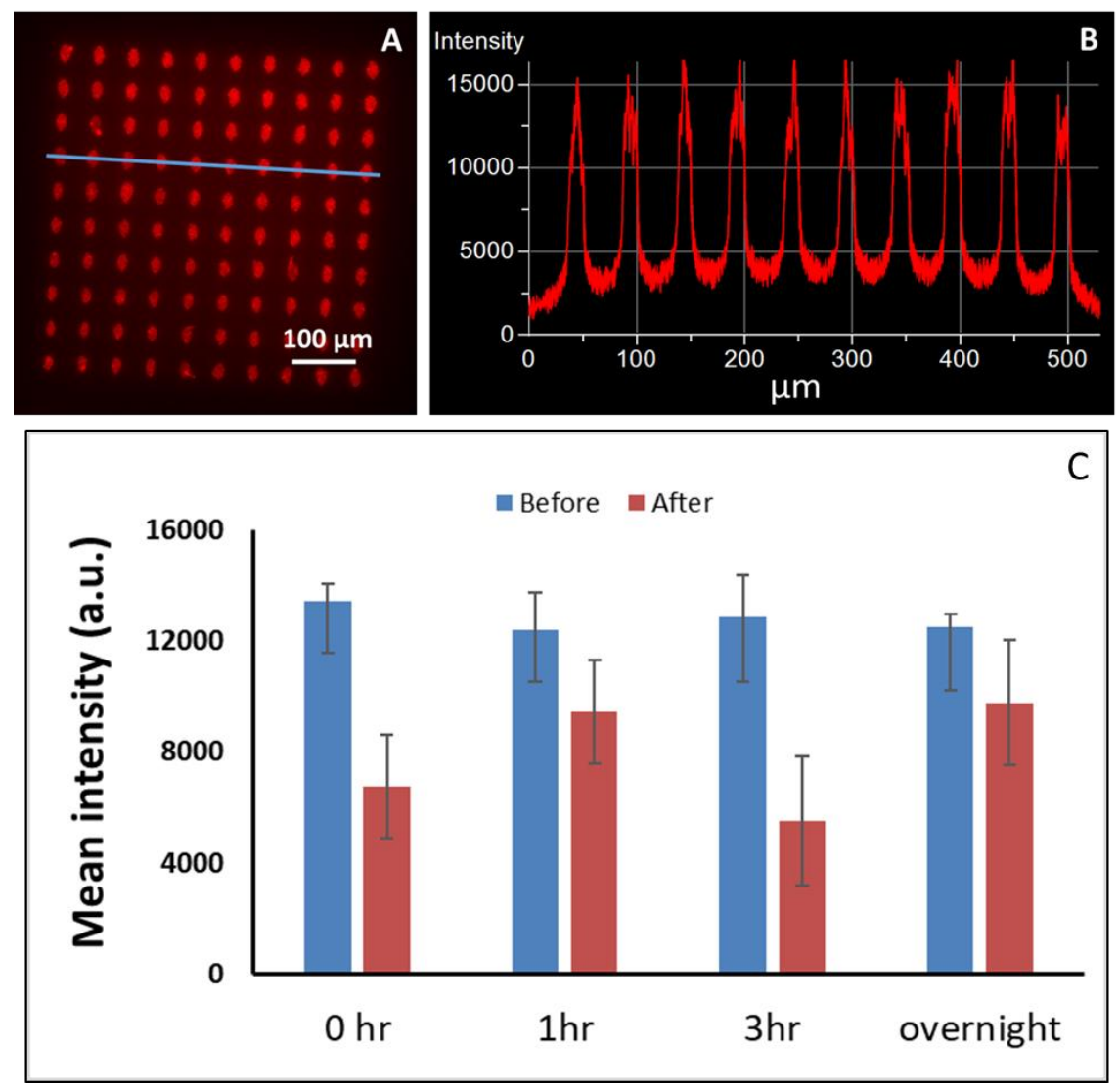

Figure S12. Fluorescence intensity comparison with different resting time. (A) typical 10x10 pattern (B) the intensity graph of the blue line on image (A). (C) comparison graph of the mean intensity with different resting time before and after washing. For each measurements, 100 dot features were evaluated, one standard deviation is given as error bars. The " $0 \mathrm{hr}$ " data point refers to immediate washing after lithography takes place, so including printing and sample transfer the protein solution would have around 5 to 10 minutes of reaction time in these cases before the ink is removed by washing. 

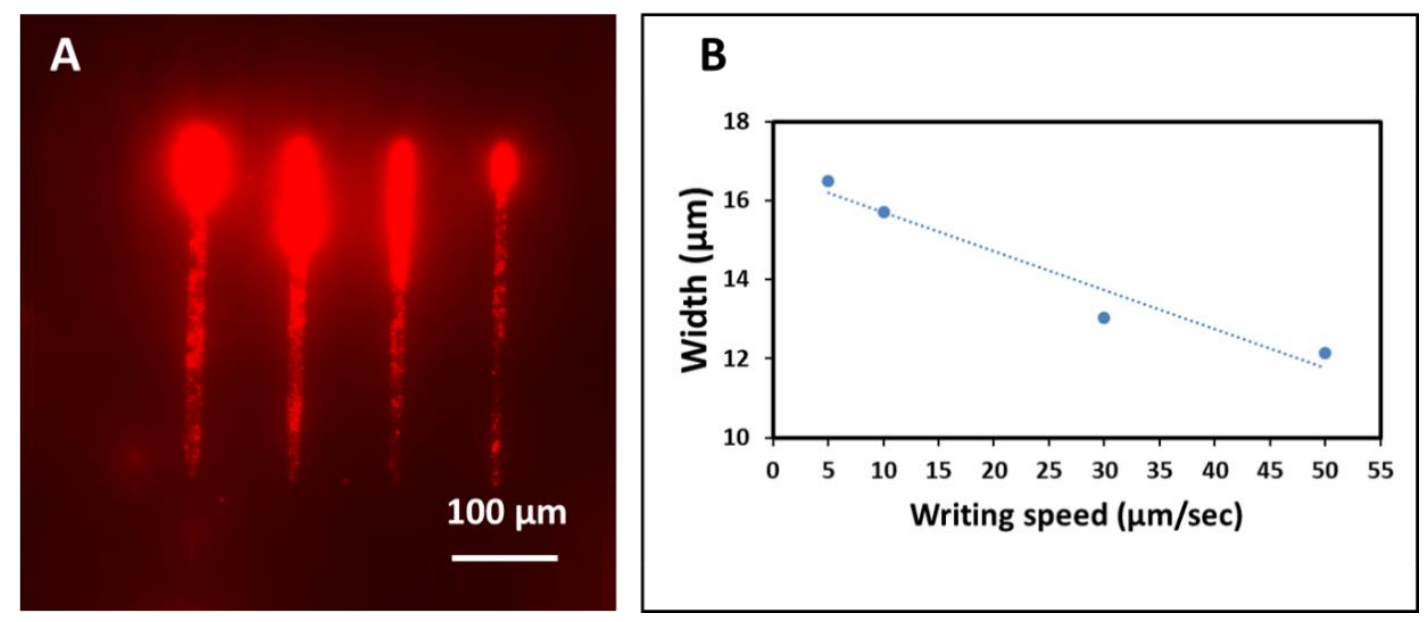

Figure S13. Line pattern with different writing speed by $\mu \mathrm{CS}$ on amine reactive polymeric dip coated substrate (A). The writing speed increased from 5 to $50 \mu \mathrm{m} / \mathrm{sec}$ (left to right on image A). The broadening at the end of the lines is caused by dragging of excess ink by the microchannel cantilever, therefore it becomes in particular prominent at low writing speeds, when more ink is deposited overall. To avoid this, higher writing speeds are preferred in generation of high resolution / high fidelity patterns.

(B) The plot of line width against writing speed reveals a linear relation $(y=-0,0984 x+16,682)$. 